\title{
Chronic Conditions and Behavioural Change Approaches to Medication Adherence: Rethinking Clinical Guidance and Recommendations
}

This article was published in the following Dove Press journal: Patient Preference and Adherence

\author{
Simon Read (D) \\ James Morgan (iD ${ }^{2}$ \\ David Gillespie $\mathbb{D D}^{3}$ \\ Claire Nollett $\mathbb{D}^{3}$ \\ Marjorie Weiss (1D ${ }^{4}$ \\ Davina Allen (D) \\ Pippa Anderson (1D ${ }^{5}$ \\ Heather Waterman (D) ${ }^{\prime}$ \\ 'School of Healthcare Sciences, Cardiff \\ University, Cardiff, Wales, UK; ${ }^{2}$ Cardiff \\ and Vale University Health Board, Cardiff, \\ Wales, UK; ${ }^{3}$ Centre for Trials Research, \\ Cardiff University, Cardiff, Wales, UK; \\ ${ }^{4}$ School of Pharmaceutical Sciences, \\ Cardiff University, Cardiff, Wales, UK; \\ ${ }^{5}$ Swansea Centre for Health Economics, \\ Swansea University, Swansea, Wales, UK
}

Correspondence: Simon Read

Cardiff University, Room 12.14, Eastgate

House, 35-43 Newport Road, Cardiff

CF24 OAB, Wales, UK

Tel +442920688930

Email readsm@cardiff.ac.uk

\begin{abstract}
Patient adherence to medication is an ongoing concern for clinicians, obfuscating treatment efficacy and resulting in wastage of medicine, reduced clinical benefit, and increased mortality. Despite this, procedural guidance on how clinicians should best engage patients regarding their medicine-taking is limited in the United Kingdom. Adherence for chronic conditions is notably complex, requiring clear education, communication, and behavioural shifts to initiate and sustain daily regimens successfully. This article explores current clinician guidance on assuring patient adherence to medication within the National Health Service, comparing it to that provided for healthcare workers in the field of behavioural change. Outlining the inertia of the former and the progress of the latter, we consider what steps should be taken to address this deficit, including greater focus on patient concerns, as well as knowledge translation for healthcare professionals in future adherence research. Current United Kingdom clinical guidance for assuring patient adherence is largely outdated based on inconclusive evidence for best practice. However, efforts to encourage behavioural change in the public health setting demonstrate evidence-based success. Integrating knowledge generated around adherence behaviour and the practical application of adherence and behavioural change research, as well as funding for longer-term studies with a focus on clinical outcomes, may help to solidify the NICE guidance on adherence and further progress the field. This would require close involvement from patient groups and networks informing ethical aspects of study design and clinical implementation.
\end{abstract}

Keywords: adherence, chronic conditions, behavioural change, clinical guidance, knowledge translation

\section{Introduction}

The 2003 World Health Organisation report into medication adherence highlighted that in developed countries only around $50 \%$ of medication was taken as prescribed, resulting in wastage of medicine, reduced clinical benefit and increases in avoidable mortality. ${ }^{1}$ In the United Kingdom, similar figures are reported with only $16 \%$ of patients prescribed new medicine taking it as intended, experiencing no problems and receiving as much information as they need. ${ }^{2}$ Consequently, 10 days after starting a prescription, a third of patients are already non-adherent. ${ }^{2}$

While considerable research and policy effort has been applied to this issue, progress towards a compelling understanding of the problem has been slow. Taking medication for chronic conditions is widely acknowledged as a complex issue, requiring mitigating efforts from both patients and healthcare professionals over the 
lifespan of treatment. ${ }^{1}$ However, much of the guidance offered to clinicians on best practice is either difficult to access or limited in scope. ${ }^{3}$

As with most clinical practice guidelines across the world, the United Kingdom's evidence-based guidance is provided by a centralised organisation; the National Institute for Health and Care Excellence (NICE). The main role of NICE is to establish balance between the benefits and costs of providing healthcare within the National Health Service (NHS) and the wider social care setting; people's needs for such services; and the desirability of promoting innovation within them. ${ }^{4}$ Again, as with similar organisations in other countries such as the United States, the purpose of this overarching advice is to enable a large health service comprised of numerous linked, independent bodies to provide high-quality care. NICE also undertakes Technology Appraisals which focus on the use of new and existing medicines, products and treatments in the NHS. ${ }^{4}$ However, the way clinical guidance is presented, particularly in relation to issues of medication adherence, can limit its practical application.

The strategy provided to NHS healthcare professionals addressing individual adherence remains relatively oblique, while the wider direction of adherence research and its translation to the clinical environment is also lacking. This article explores the existing NICE clinical guidelines for medication adherence in chronic conditions, looking to identify the root of these strategic deficits. In doing so, we will compare this limited guidance to that offered in the related field of behavioural change, where considerable progress has been made.

\section{Guidance on Medication Adherence}

Guidance on adherence is provided through NICE documentation specifically addressing the issue (CG76), as well as being briefly referred to within condition-specific guidelines. Condition-specific guidelines offer limited advice for clinicians, predominantly indicating that discussions around patient medicine-taking should be integrated into clinical consultations, the need for patient education, information provision and understanding of their condition, as well as communication and support channels. However, precisely what these discussions should entail and the theoretical framework influencing them remain unclear. ${ }^{5-7}$

Instead, for further detail, clinicians are largely directed to the wider CG76 guidance on adherence. Last updated in 2009, the CG76 document theoretically frames adherence through a binary model of intentional and unintentional adherence issues. ${ }^{8}$ The intentional and unintentional concept of adherence behaviour was developed to outline more common examples of how patients do not take their medication, first being formally proposed in $2002 .{ }^{9}$ Unintentional adherence issues are defined by patient passivity provoking issues with the maintenance of their regimen. This could relate to difficulties with prescription refills, inadequate understanding of treatment plans, poor education, or other factors such as physical difficulties in administration. However, intentional adherence issues are characterised by an active patient effort to modify or reject their treatment plan, reflecting a decision-making process incorporating competing and conflicting factors. ${ }^{10}$ This element of the adherence conundrum has attracted most attention, with evidence suggesting that it is influenced by a range of factors such as medication side effects, inherent beliefs about medication or the health system, and the patient's perception of their condition. ${ }^{10}$

While the theoretical analysis of intentional and unintentional adherence has granted a more nuanced understanding of patient behaviour, the limitations of the approach have become increasingly clear over the last 15 years. A major problem is the theoretical overlap between intentional and unintentional actions, both of which can contribute to the same behavioural outcome. Forgetfulness, for instance, could arise from unintentional memory issues and/or an intentional motivation to deprioritise treatment. ${ }^{11}$ Furthermore, this approach overwhelmingly stems from a psychological foundation, limiting contributory social factors affecting adherence. More importantly, though, this theoretical gap has been unhelpful in identifying adherence interventions that address these problems.

\section{Inconclusive Evidence on Adherence Best Practice}

A recent review of 49 randomised clinical trials identified several techniques to improve adherence. ${ }^{12}$ These include patient education, medication regimen management, medication-taking reminders, motivational interviewing to improve cognitive behaviour, and consultations with clinical pharmacists for chronic disease co-management. ${ }^{12}$ Each of these techniques has been evidentially successful in the research setting, offering greater granularity on how clinicians can respond to adherence issues. However, although the NICE guidance on medicines adherence acknowledges the existence of these techniques, the document predominantly emphasises the inconclusiveness of such interventions and recommends their use only when specific needs have been identified. ${ }^{8}$ 
The extent to which this problematises adherence assessments during clinical practice is debatable. Taking the chronic eye condition of glaucoma as an example, as with much condition-specific guidance, NICE mention patient education, information provision, and access to support channels. ${ }^{7}$ However, when or how this should be integrated into the care pathway, who is best placed to perform such activities, and what these activities should entail remain opaque.

This lack of an overarching view on best practice arguably contributes to an absence of adherence checks within standard care. ${ }^{13}$ Rather than attempt to address difficult-toidentify adherence issues with inconclusive interventions, the default pathway for many clinicians may be to assume treatment inefficacy. ${ }^{13}$ Consequently, patients can often be provided additional treatment for conditions they are struggling to manage for other complex reasons. ${ }^{14}$ This also has an economic impact for the NHS, with a recent systematic review reporting that across 14 disease groups lower levels of adherence were generally associated with higher treatment costs. That said, the quality of these studies has been critiqued, with few demonstrating the highest research standards. ${ }^{15}$

\section{Behavioural Change Guidance and the COM-B Approach}

Although their links are not formalised within NICE guidance, the issues of behavioural change and medication adherence for chronic conditions are underpinned by many common concerns. A synthesis of qualitative research covering patient views has concluded that many actively resist taking medicines based on concerns over their necessity and effectiveness. ${ }^{16}$ Furthermore, the complexity associated with these responses to adherence has been shown to emanate from a range of behavioural dimensions, including psychological reactance. ${ }^{17}$ Patients being prescribed new long-term medication require similar levels of support, encouragement, and education to those making other long-lasting lifestyle changes. This requirement is greater still where conditions are asymptomatic and treatments are to prevent deterioration, as with daily use of statins for hypertension or eye drops for glaucoma.

The NICE documents on individual (PH49) and more general (PH6) approaches to behavioural change target issues such as dieting, encouraging physical exercise, and reducing health-damaging behaviours. ${ }^{18,19}$ Much like adherence behaviours, the root of these issues is complex and variable for different patients. Yet, these recommendations demonstrate greater strategic direction about which individual and structural approaches may aid meaningful change in the area. These include development of locally specific policies and strategies, provision of training to staff, the use of proven, evidence-based intervention techniques, and interlinkage between national bodies, such as Royal Colleges, and local organisations delivering interventions. Such efforts have arguably enabled the field of behavioural change to advance, with long-term patient involvement and effective knowledge translation being a key force.

The Capability, Opportunity and Motivation (COM-B) model of behaviour ${ }^{20,21}$ is the central theoretical framework for these NICE recommendations. The model is based on health psychology but also accounts for structural, social, and environmental factors, making it applicable to many situations where behavioural change is required. Offering greater granularity than the intentional/unintentional model of adherence outlined in the NICE guidance, the categories of COM-B fall into six distinct types: psychological and/or physical capability; physical or social opportunity; reflective and/or automatic motivation. In relation to medication adherence, it is proposed that physical capability could apply to issues of dexterity that may hinder medicinetaking, as well as ability to adapt to changes in lifestyle. ${ }^{22}$ Psychological capability, however, refers to a patient's understanding of their condition, as well as how their medication influences it. This could also include issues of cognitive impairment and memory issues. ${ }^{22}$ Physical and social opportunity comprises access issues such as availability of medication, packaging problems or regimen complexity, as well as religious or social beliefs, or the stigma associated with conditions. ${ }^{22}$ Reflective and automatic motivation involve patient perception of their illness, beliefs around its treatment and the likely clinical outcomes, as well as education provision for patients and issues associated with depression or changing mood states. ${ }^{22}$

Research within the field has expanded quickly, with the development of a comprehensive list of behaviour change techniques weighted by their evidence-based effectiveness. ${ }^{19}$ Examples where techniques have been shown to be successful include the use of self-monitoring in the promotion of physical exercise or healthy eating, and goal setting for smoking cessation $^{22}$ (see Table 1 for further examples, including studies on adherence). Gathering knowledge on factors influencing adherence should be conducted within a similar framework encouraging long-term patient involvement and informed, evidence-based interventions. However, prior to this 
Table I Examples of Studies Using COM-B Model with Health Behaviours

\begin{tabular}{|l|l|}
\hline Reference & $\begin{array}{l}\text { Targeted Health } \\
\text { Behaviour }\end{array}$ \\
\hline $\begin{array}{l}\text { Michie S, Abraham C, Whittington C et al } \\
\text { (2009): Effective techniques in healthy eating } \\
\text { and physical activity interventions: a meta- } \\
\text { regression. Health Psychology, 28 (6): 690-70I. }\end{array}$ & $\begin{array}{l}\text { Healthy eating/ } \\
\text { physical activity }\end{array}$ \\
\hline $\begin{array}{l}\text { Lorencatto F, West R, Michie S (20I2): } \\
\text { Specifying evidence-based behavior change } \\
\text { techniques to aid smoking cessation in } \\
\text { pregnancy. Nicotine \& Tobacco Research, 14 (9): } \\
\text { I0I9-I026. }\end{array}$ & Smoking \\
\hline $\begin{array}{l}\text { Ellis K, Pears S, Sutton S (20I9): Behavioural } \\
\text { analysis of postnatal physical activity in the UK } \\
\text { according to the COM-B model: a multi- } \\
\text { methods study. BMJ Open, } 9 \text { (8). }\end{array}$ & Physical activity \\
\hline $\begin{array}{l}\text { Barker F, Atkins L, de Lusignan S (20I6): } \\
\text { Applying the COM-B behaviour model and } \\
\text { behaviour change wheel to develop an } \\
\text { intervention to improve hearing-aid use in } \\
\text { adult auditory rehabilitation. International } \\
\text { Journal of Audiology, 55 (3): S90-S98. }\end{array}$ & \\
\hline $\begin{array}{l}\text { Felix B, Guerreiro MP, Cavaco A et al (20I9): } \\
\text { Development of a Complex Intervention to } \\
\text { Improve Adherence to Antidiabetic } \\
\text { Medication in Older People Using an } \\
\text { Anthropomorphic Virtual Assistant Software. } \\
\text { Frontiers of Pharmacology, I0: 680. }\end{array}$ & adherence \\
\hline $\begin{array}{l}\text { Herber O, Atkins L, Stork S et al (20I8): } \\
\text { anhancing self-care adherence in patients with } \\
\text { study). BMJ Open, 8 (9). }\end{array}$ & \\
\hline
\end{tabular}

a systemic reconsideration of adherence is required, accounting for some of the behavioural, structural, and theoretical problems outlined above.

\section{Discussion}

Healthcare professionals and researchers in the NHS are cursorily aware of the systemic issues provoking variation in standard care. Many health settings are busy with an unpredictable level of case complexity limiting opportunities for patient communication and interaction. In these situations, unfamiliar and poorly defined processes are more likely to be sacrificed than those felt to be clinically critical. Based on the NICE guidance for adherence where there are limited overarching mechanisms to guide clinical behaviour, adoption of strategies to enhance adherence behaviour in clinic is understandably limited. Comparable issues have been identified in the clinical practice guidelines of the United States, where advice on medication adherence is similarly oblique offering "vague strategies, with few specifics". ${ }^{23}$ Additionally, the inconclusive evidence on specific adherence interventions suggests that any techniques clinicians in either country might deploy may have limited effect.

Improving adherence will have health-related benefits for patients and enable better use of healthcare resources. The linkage of available information on medication adherence with theories emerging from social science, ${ }^{24}$ comprehensive theories of behavioural change and guidance on specific conditions would help to improve clinician engagement with their patients' medicine-taking. As well as offering a more developed model of the factors influencing adherence, the growth of knowledge around intervention techniques would support better-informed decisions on those best suited for specific patients, which healthcare professional is best placed to perform them and at what stages they should be utilised. While individualised behavioural interventions have not yet been developed, the data gathered around those elements which work for certain patients at certain times can foster progress in this direction. Additionally, the integration of overlapping and related NICE documentation would also address clinicianreported issues of information overload. ${ }^{25}$

This updating and restructuring of guidance and recommendations should be carried out alongside a theoretical and methodological rethinking of adherence itself. The definition outlined within current NICE guidance has been theoretically superseded. A more contemporary taxonomy of medication adherence suggests that it should be considered over the lifespan of the prescription so that specific issues can be addressed in a phased manner. These phases include medication initiation, implementation, persistence, and discontinuation (see Table 2 for definitions). ${ }^{26}$ Thus, a patient initiating a new regimen may be more prone to difficulties arising from physical opportunities such as the availability of a prescription, or a lack of patient education. By contrast, another patient may be more likely to encounter motivational issues persisting with their medication much later into the prescription's lifespan.

We recommend future research in the field to continue testing appropriate, evidence-based behaviour change techniques within a theoretical framework that accounts 
Table 2 Adherence Taxonomy (2012) Definitions

Adherence to medications is defined as the process by which patients take their medications as prescribed, composed of initiation, implementation, persistence and discontinuation.

Initiation occurs when the patient takes the first dose of a prescribed medicine.

Implementation is the extent to which a patient's actual dosing corresponds to the prescribed dosing regimen, from initiation until the last dose.

Persistence is the length of time between initiation and the last dose, which immediately precedes discontinuation.

Discontinuation occurs when the patient stops taking their prescribed medicine.

Note: Data from Vrijens et al. A new taxonomy for describing and defining adherence to medications. $\mathrm{Br}$ J Clin Pharmacol. 2012;73(5):69I-705. ${ }^{26}$

for factors influencing adherence. Importantly, any such studies should recognise that these factors are likely to vary over time and will require ongoing patient support and education, as well as consideration as to which healthcare professionals may be best placed to provide this.

For this to succeed, data collection methods also need to be addressed. Many adherence studies have focused on the short-term aspects of treatment primarily because of limited funding for longitudinal studies. Consequently, there has been a tendency to study proxy clinical outcomes, given that these are more easily measurable in limited time frames. For instance, research into adherence for glaucoma eye drops has focused on the primary outcome of eye pressure reduction rather than the prevention of vision loss and condition severity. ${ }^{27}$ Numerous studies have embraced the initiation and implementation phases of a newly diagnosed medicine but have lacked the resources to track this over long-term treatment of chronic conditions.

Observations are confounded by the poor reliability of adherence data drawn from patient self-reporting, prescription refills or event monitoring systems that record actions such as the opening and closing of a pillbox, but not the taking of the medicine. These types of data collection closely mirror actual behaviour but remain proxy measures that are far from ideal. Developments in technology may enable future measures that more accurately account for the actual administration of medicine and the resource impact of adherence issues; the pursuit of these should also be a key priority.

Alongside the need for more accurate measurement and a clearer understanding of what influences variability in adherence over time, both long-term patient involvement and effective translation of research into healthcare professional practice are paramount. Patient choices regarding their health and how their adherence is monitored are key to unpicking behavioural fluctuations and subsequently how best to individually address them. Future studies looking to examine adherence behaviours should consider patient perspectives in both study design and implementation, ensuring that ethical concerns in relation to monitoring of medicinetaking are sensitively negotiated in advance. Aside from this, establishing adherence assessments and evidence-based interventions within clinical consultations also requires the buy-in of healthcare professionals. Re-emphasising and clarifying the value of such interactions within NICE guidance would be a useful step, but ensuring clinicians are able to execute them through clearer support and advice will require a multifaceted approach addressing the issues outlined above.

\section{Acknowledgments}

The research leading to this article was funded by Health and Care Research Wales.

\section{Disclosure}

The authors report no conflicts of interest in this work, financial or otherwise.

\section{References}

1. Sabaté E. Adherence to long-term therapies: evidence for action. 2003. Available from: https://www.who.int/chp/knowledge/publications/ adherence full report.pdf?ua=1. Accessed November 25, 2019.

2. Picton C, Wright H. Medicines Optimisation: Helping Patients to Make the Most of Medicines. London: Royal Pharmaceutical Society; 2013.

3. Nieuwlaat R, Wilczynski N, Navarro T, et al. Interventions for enhancing medication adherence. Cochrane Database Syst Rev. 2014;11: CD000011. doi:10.1002/14651858.CD000011.pub4

4. National Institute for Health and Care Excellence. NICE guidance. 2019. Available from: https://www.nice.org.uk/about/what-we-do/ourprogrammes/nice-guidance. Accessed January 16, 2020.

5. National Institute for Health and Care Excellence. Hypertension in adults: diagnosis and management. 2019. Available from: https://www. nice.org.uk/guidance/ng136. Accessed January 16, 2020.

6. National Institute for Health and Care Excellence. Type 1 diabetes in adults: diagnosis and management. 2016. Available from: https://www. nice.org.uk/guidance/ng17/chapter/Patient-centred-care. Accessed January 16, 2020.

7. National Institute for Health and Care Excellence. Glaucoma: diagnosis and management. 2017. Available from: https://www.nice.org.uk/ guidance/ng81/evidence/full-guideline-pdf-4660991389. Accessed November 25, 2019.

8. National Institute for Health and Care Excellence. Medicines adherence: involving patients in decisions about prescribed medicines and supporting adherence. 2009. Available from: https://www.nice.org.uk/ guidance/cg76. Accessed November 25, 2019. 
9. Wroe A. Intentional and unintentional nonadherence: a study of decision making. J Behav Med. 2002;25(4):355-372. doi:10.1023/ A:1015866415552

10. Lowry K, Dudley T, Oddone E, et al. Intentional and unintentional nonadherence to antihypertensive medication. Ann Pharmacother. 2005;39:1198-1203. doi:10.1345/aph.1E594

11. McHorney C, Spain C. Frequency of and reasons for medication non-fulfillment and non-persistence among American adults with chronic disease in 2008. Health Expect. 2010;14:307-320. doi:10.1111/j.1369-7625.2010.00619.x

12. Kini V, Ho P. Interventions to improve medication adherence: a review. J Am Med Assoc. 2018;320(23):2461-2473. doi:10.1001/ jama.2018.19271

13. Riekert K. Integrating regimen adherence assessment into clinical practice. In: O'donohue W, Levensky E, editors. Promoting Treatment Adherence: A Practical Handbook for Health Care Providers. London: Sage; 2006:17-34.

14. Lam W, Fresco P. Medication adherence measures: an overview. Biomed Res Int. 2015;2015:1-12. doi:10.1155/2015/217047

15. Cutler RL, Fernandez-llimos F, Frommer M, et al. Economic impact of medication non-adherence by disease groups: a systematic review. BMJ Open. 2018;8:e016982. doi:10.1136/bmjopen-2017-016982

16. Pound P, Britten N, Morgan M, et al. Resisting medicines: a synthesis of qualitative studies of medicine taking. Soc Sci Med. 2005;61 (1):133-155. doi:10.1016/j.socscimed.2004.11.063

17. De las Cuevas C, De Leon J. Development and validation of the patient's health belief questionnaire on psychiatric treatment. Patient Prefer Adherence. 2019;13:527-536. doi:10.2147/PPA.S201144

18. National Institute for Health and Care Excellence. Behaviour change: individual approaches. 2014. Available from: https://www.nice.org. uk/guidance/ph49. Accessed July 31, 2019.
19. Institute for Health and Care Excellence. Behaviour change: general approaches. 2007. Available from: https://www.nice.org.uk/guidance/ ph6. Accessed July 31, 2019.

20. Michie S, van Stralen M, West R. The behaviour change wheel: a new method for characterising and designing behaviour change interventions. Implement Sci. 2011;6(42):1-11. doi:10.1186/1748-5908-6-42

21. Michie S, Richardson M, Johnston M, et al. The behaviour change technique taxonomy (v1) of 93 hierarchically clustered techniques: building an international consensus for the reporting of behaviour change interventions. Ann Behav Med. 2013;46(1):81-95. doi:10.1007/s12160-013-9486-6

22. Jackson C, Eliasson L, Barber N, et al. Applying COM-B to medication adherence: a suggested framework for research and interventions. Eur Health Psychol. 2014;16(1):7-17.

23. Ruppar T, Dobbels F, Lewek P, et al. Systematic review of clinical practice guidelines for the improvement of medication adherence. Int J Behav Med. 2015;22:699-708. doi:10.1007/s12529-015-9479-x

24. May C, Eton D, Boehmer K, et al. Rethinking the patient: using burden of treatment theory to understand the changing dynamics of illness. BMC Health Serv Res. 2014;14:281. doi:10.1186/1472-696314-281

25. Carthey J, Walker S, Deelchand V, et al. Breaking the rules: understanding non-compliance with policies and guidelines. $\mathrm{Br} \mathrm{Med} J$. 2011;343:d5283. doi:10.1136/bmj.d5283

26. Vrijens B, De Geest S, Hughes D, et al. A new taxonomy for describing and defining adherence to medications. $\mathrm{Br} \mathrm{J}$ Clin Pharmacol. 2012;73(5):691-705. doi:10.1111/bcp.2012.73.issue-5

27. Sleath B, Blalock S, Covert D, et al. The relationship between glaucoma medication adherence, eye drop technique, and visual field defect severity. Ophthalmology. 2011;118(12):2398-2402. doi:10.1016/j.ophtha.2011.05.013
Patient Preference and Adherence

\section{Publish your work in this journal}

Patient Preference and Adherence is an international, peer-reviewed, open access journal that focuses on the growing importance of patient preference and adherence throughout the therapeutic continuum. Patient satisfaction, acceptability, quality of life, compliance, persistence and their role in developing new therapeutic modalities and compounds to optimize clinical outcomes for existing disease states are major areas of interest for the journal. This journal has been accepted for indexing on PubMed Central. The manuscript management system is completely online and includes a very quick and fair peer-review system, which is all easy to use. Visit http:// www.dovepress.com/testimonials.php to read real quotes from published authors. 